\title{
Corrigendum: Genome-wide association study identifies multiple loci associated with both mammographic density and breast cancer risk
}

Sara Lindström, Deborah J. Thompson, Andrew D. Paterson, Jingmei Li, Gretchen L. Gierach, Christopher Scott, Jennifer Stone, Julie A. Douglas, Isabel dos-Santos-Silva, Pablo Fernandez-Navarro, Jajini Verghase, Paula Smith, Judith Brown, Robert Luben, Nicholas J. Wareham, Ruth J.F. Loos, John A. Heit, V. Shane Pankratz, Aaron Norman, Ellen L. Goode, Julie M. Cunningham, Mariza deAndrade, Robert A. Vierkant, Kamila Czene, Peter A. Fasching, Laura Baglietto, Melissa C. Southey, Graham G. Giles, Kaanan P. Shah, Heang-Ping Chan, Mark A. Helvie, Andrew H. Beck, Nicholas W. Knoblauch, Aditi Hazra, David J. Hunter, Peter Kraft, Marina Pollan, Jonine D. Figueroa, Fergus J. Couch, John L. Hopper, Per Hall, Douglas F. Easton, Norman F. Boyd, Celine M. Vachon \& Rulla M. Tamimi

Nature Communications 5:5303 doi: 10.1038/ncomms6303 (2014); Published 24 Oct 2014; Updated 9 Sep 2015

The financial support for this Article was not fully acknowledged. The Acknowledgements should have included the following: This study was supported by CA173785 from the National Institutes of Health. 